EXTENDED REPORT

\title{
18 Years' experience with high dose rate strontium-90 brachytherapy of small to medium sized posterior uveal melanoma
}

\author{
R van Ginderdeuren, E van Limbergen, W Spileers
}

Br J Ophthalmol 2005;89:1306-1310. doi: 10.1136/bjo.2005.068460

See end of article for authors' affiliations

Correspondence to: Rita van Ginderdeuren, Department of Ophthalmology, Kapuciinenvoer 33, UZ Leuven, B3000 Leuven, Belgium; rita. vanginderdeuren@uz. kuleuven.ac.be

Accepted for publication 6 May 2005 adiation related complications, visual acuity, enucleation rate, and Aim: To analyse local tumour control, radiation related complications, visual acuity, enucleation rate, and
survival after brachytherapy of small to medium sized choroidal melanoma (CM) with a high dose rate (HDR) strontium-90 (Sr-90) applicator.

Methods: From 1983 until 2000, 98 eyes with CM were treated with Sr-90 brachytherapy. The main outcome measures were actuarial rates of the patients' survival, ocular conservation rate, tumour regression, complication rates, and preservation of visual acuity. End point rates were estimated using Kaplan-Meier analysis.

Results: The median follow up time was 6.7 years $(0.5-18.8$ years). Actuarial melanoma free patient survival rate was $85 \%$ (SE $4.8 \%$ ) after 18 years. Actuarial rate of ocular conservation and complete tumour regression was $90 \%$ (SE 3.8\%) after 15 years. In 93\% local tumour control was achieved, 88\% showed a stable scar. Recurrence of the tumour on the border caused enucleation of six eyes (7\%). In three cases $(4 \%)$ retinal detachment was the end point. No cases of optic atrophy or of sight impairing retinopathy outside the treated area were found. Actuarial rate of preservation of visual acuity of $1 / 10$ was $65 \%$ at 5 years and $45 \%$ at 15 years of follow up (SE $5.9 \%$ and $8.8 \%$ ).

Conclusions: Sr-90 brachytherapy is as effective as iodine or ruthenium brachytherapy for small to medium sized CM but causes fewer complications. The preservation of vision is better than with all other described radioisotopes. HDR Sr-90 brachytherapy can therefore safely be recommended for small to medium sized $C M$.
M ultiple techniques are used for the treatment of uveal melanoma and different radioisotopes are used for brachytherapy. ${ }^{1-6}$ We have used the high dose rate (HDR) strontium-90 (Sr-90) applicator since 1983. The main difference between Sr-90 and the other applicators is the very high dose rate ( $10200 \mathrm{cG} /$ hour) of $\beta$ irradiation: this reduces the treatment to one session of 2-5 hours. Because Sr-90 has a half life of 28.5 years, we have been able to use the same applicator for the past 21 years. Our preliminary results were reported in $1998 .^{78}$ In this paper we review our results on a larger group of patients with a longer follow up time.

\section{PATIENTS AND METHODS}

Patient characteristics

From 1983 to 2000, 98 eyes with CM were treated with HDR Sr-90 brachytherapy. Eighty nine patients were treated with Sr-90 as first treatment and are included in the study. The other nine patients were treated first with another treatment and are excluded. The patients comprised 49 women and 40 men, with a median age of 64 years (range 29-85 years). All patients were followed for involution of the tumour and complications of the therapy until December 2003 or until death. Metastasis was proved by liver ultrasound and biochemical tests. The exact date and cause of death were known for all patients.

\section{Tumour characteristics}

Before treatment, the tumour had a median maximum basal diameter of $9.5 \mathrm{~mm}$ (range 6-15.3 $\mathrm{mm}$ ). The maximum height of the tumour before treatment was measured by ultrasound and ranged between $1.2 \mathrm{~mm}$ and $8.4 \mathrm{~mm}$ with a median of $4.1 \mathrm{~mm}$. Sixteen tumours $(18 \%)$ belong to the group of small tumours (thickness less than $3 \mathrm{~mm}$ and diameter less than $10 \mathrm{~mm}$ ) and 73 to medium size tumours.

\section{Treatment protocol}

The exact characteristics of the Sr-90 applicator, used in our group, and the treatment protocols have already been fully described. ${ }^{7}$ An epibulbar surface applicator (SIAQ 7321) produced by the Amersham Company was modified by removing the shaft (figs 1, 2). It was loaded with $370 \mathrm{MBq}$ (10 mCi) Sr-90 at the concave side and shielded with $0.1 \mathrm{~mm}$ platinum. In 1983 the dose rate at the applicator's surface was measured at $7.2 \mathrm{cGy} / \mathrm{s}$. The dose delivered in the beginning of the study was $75 \mathrm{~Gy}$ at the tumour apex (14 patients), corresponding to a scleral dose between $299 \mathrm{~Gy}$ and $710 \mathrm{~Gy}$. Because of recurrence of a small thin tumour and success of a tumour of $8 \mathrm{~mm}$ thickness, we believed that it was more important to irradiate the base to a sufficient large dose. From June 1990 we treated 59 patients with a scleral dose of $600 \mathrm{~Gy}$, administered in less than 2 hours. Because of the decay of Sr-90 the required time exceeded 3 hours in 1999 and we decided to lower this scleral dose to 450 Gy ( 12 patients). The size of the applicator was not sufficient for covering the whole tumour in four patients, and a shift of the applicator was performed in one treatment (total scleral dose $800 \mathrm{~Gy}$ ). Sixty six patients were treated with one session. For insufficient regression or suspected recurrence at the tumour border, 18 patients were treated at a later point with a second session of Sr-90, and five patients with a third session. For very small suspicious areas of the tumour xenon arc treatment was applied in nine patients and

Abbreviations: $C M$, choroidal melanoma; $\mathrm{HDR}$, high dose rate; $\mathrm{Sr}-90$, strontium-90 
A

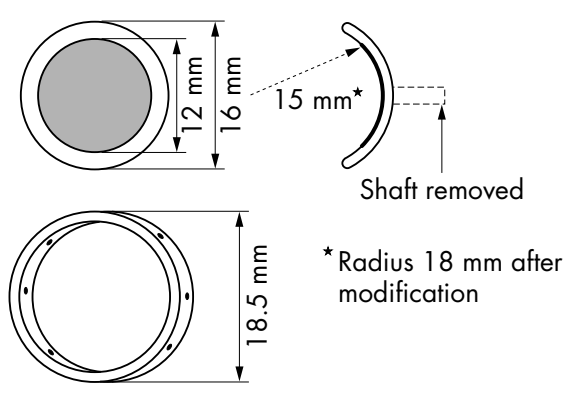

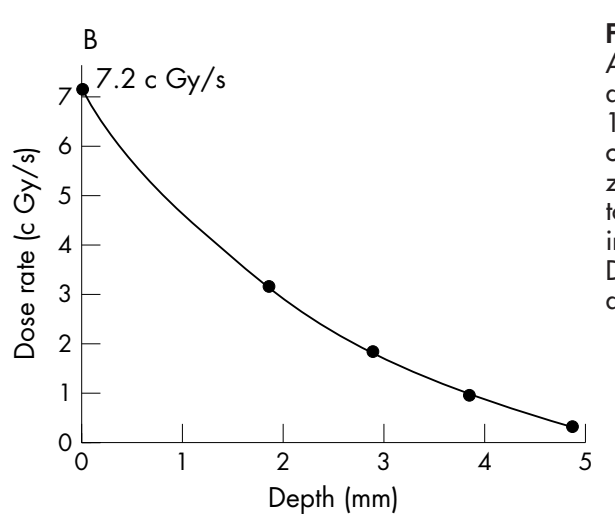

Figure 1 (A) Modification of the Amersham SIAQ 7321 Sr-90 applicator. After removing the shaft, a $16 \mathrm{~mm}$ diameter applicator was obtained with a $12 \mathrm{~mm}$ radioactive zone. The perforated stainless steel ring to fix the applicator to the sclera has an internal diameter of $16.5 \mathrm{~mm}$. (B) Depth dose curve of the $\mathrm{Sr}-90$ applicator. in the latter part of the study diode laser in three patients, following the protocol of transpupillary thermotherapy (TTT).

\section{Analyses}

Actuarial rates of patient melanoma free survival, tumour control, ocular conservation, and preservation of visual acuity of $1 / 10$ were estimated using Kaplan-Meier analysis (Statistica, Statsoft, Inc, USA).

The study was approved by the ethics committee of UZLeuven.

\section{RESULTS}

\section{Survival rate}

The median follow up time is 6.7 years (range 0.518.8 years). The number of patients followed for $3,5,8$ and 10 years is, respectively, 78, 57, 55, and 25. At 3, 5, and 15 years post-treatment follow up, melanoma free patient survival was, respectively, 91\%, 90\%, and $85 \%$ (SE 2.9\%, $3.2 \%$, and $4.8 \%$ ). Eleven patients $(12 \%)$ died of melanoma related metastatic disease between 6 months and 9 years (fig 3). Six patients died in the follow up period as a result of tumour unrelated causes.

\section{Tumour control}

Actuarial rate of tumour control after 3, 5, and 10 years was, respectively, $95 \%, 92 \%$, and $90 \%$ (SE $2.0 \%, 3.0 \%$, and $3.8 \%$ ). Local tumour control was obtained in 83 eyes (93.3\%), from which 78 regressed to a scar: naked sclera or flat pigmented choroidal rest tissue, which could not be detected by ultrasound (fig 4). The other five patients died as a result of metastases during regression of the lesion but before reaching a flat scar. Treatment failure (growth beyond control at the tumour border) caused enucleation in six patients $(7.3 \%)$. One eye presented with a second ciliary body melanoma after complete regression of macular treated

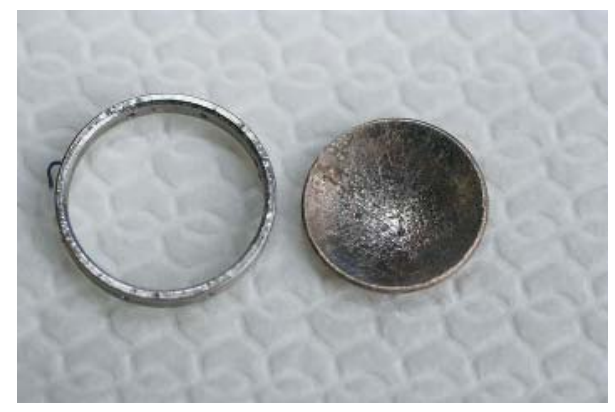

Figure 2 Picture of actual Sr-90 applicator with perforated stainless steel ring to fix the applicator to the sclera.

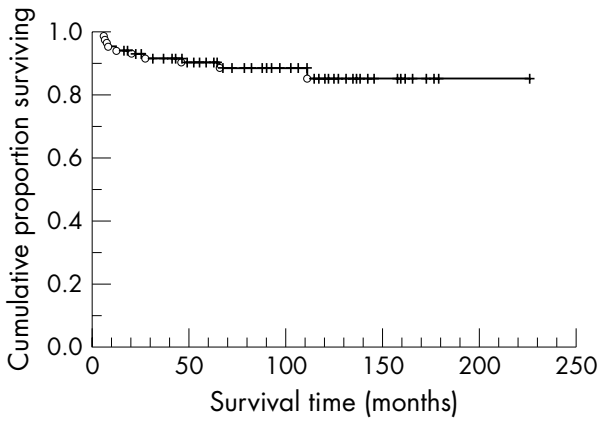

Figure 3 Kaplan-Meier melanoma free patients survival curve after $\mathrm{Sr}$ 90 treatment, expressed in months of follow up. (Survival function: 0 , complete (death by metastasis); +, censored.)

melanoma and was enucleated. No melanocytosis or connection between the two locations was found histologically. ${ }^{8}$

There was no difference in regression between the different treatment protocols. Tumour control was obtained in 13/14 patients with $75 \mathrm{~Gy}, 54 / 59$ with $600 \mathrm{~Gy}, 11 / 12$ with $450 \mathrm{~Gy}$, and $4 / 4$ with $2 \times 400 \mathrm{~Gy}$.

\section{Complication rate}

No cases of sight impairing radiation retinopathy outside the treated area were found. In $21 \%$ of cases mild radiation retinopathy was present with temporary small retinal exudates and haemorrhages; these regressed after years. An exudative retinal detachment is seen around the treated area; this regressed spontaneously, except in four patients. Persistent complications comprised retinal detachment, ${ }^{3}$ macular fibrosis, ${ }^{1}$ diplopia. $^{3}$ No painful eye, neovascular glaucoma, optic atrophy (distance tumour to optic disc was $3 \mathrm{~mm}$ minimum) or scleral melting were observed. Nontumour related surgery was performed in eight cases: vitrectomy ${ }^{4}$ for intravitreal bleeding or retinal detachment, argon laser photocoagulation for ischaemic retina because of obliteration of retinal vessels passing over the tumour, ${ }^{2}$ and diabetes. $^{2}$ Actuarial rates of ocular conservation give a cumulative survival of the eye of, respectively, 95\%, 92\%, and $90 \%$ after 3,5 , and 15 years (SR $2.3 \%, 3.0 \%$, and $3.8 \%$ ) (fig 5). No eyes were enucleated because of complications of the treatment.

\section{Visual acuity results}

The visual acuity before treatment, and at 3 years and 5 years of follow up is shown in table 1 . Actuarial rate of visual acuity of at least $1 / 10$ is $74 \%, 65 \%$, and $45 \%$ at, respectively, 3,5 , and 15 years follow up (SE $5.2 \%, 5.9 \%$, and $8.8 \%$ ), and is expressed in figure 6. More than one third of patients had at the end point a vision of more than $5 / 10 ; 58 \%$ of all our 


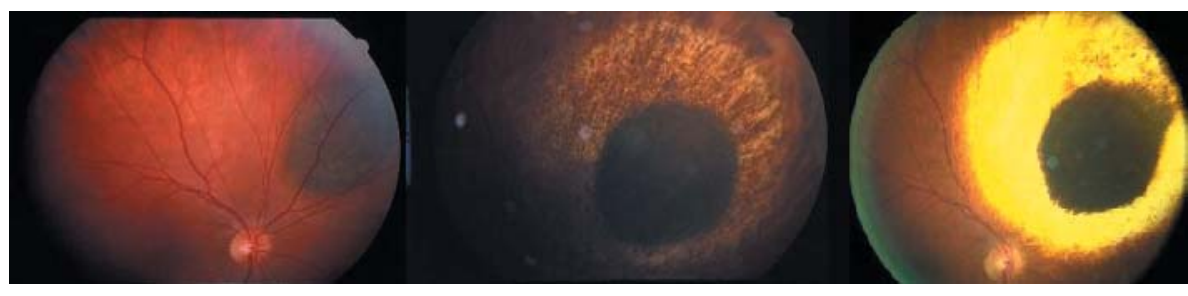

Figure 4 Picture of melanoma before treatment (left), after 6 months (centre), and after 5 years (right). The initial vision was $8 / 10$; the lesion was $8 \mathrm{~mm}$ in diameter and $3 \mathrm{~mm}$ thick (ultrasound). Strontium treatment was performed for 2 hours 36 minutes. The mass regressed during the first 6 months. After 5 years there was $3 / 10$ vision and no radiation retinopathy. No optic nerve atrophy could be detected.

patients retained vision $1 / 10$ or more after a median follow up time of 6.7 years. Only 13 of the 89 patients lost useful vision, including the enucleated eyes. From all patients with vision of $1 / 10$ or more at the time of treatment, $69 \%$ retained $1 / 10$ vision after median follow up of 6.7 years.

\section{DISCUSSION}

In this study small and medium sized CM were treated by an unconventional brachytherapy. This HDR Sr-90 treatment controlled the tumour in $90 \%$ of cases, with preservation of good vision in $45 \%$ after 15 years. The long follow up time indicates a persistent good result with survival times similar to other treatments.

Sr-90 is a $\beta$ emitter with high dose rate; because of the decay the actual dose rate of our applicator is $4.5 \mathrm{cGy} / \mathrm{s}$ or $10200 \mathrm{cGy} /$ hour. A high dose delivered at a high rate is radiobiologically very efficient in cell kill. ${ }^{2}$ We believe this explains the high rate of success with very fast shrinking of the tumour and high number of flat scars. After Sr-90 treatment the tumour shows swelling after 2 weeks. All the vessels in the irradiated field are occluded in 2 weeks, proved by fluoroangiography performed after treatment. ${ }^{7}$ During the first 6 months after treatment the tumour shrinks very quickly.

The characteristics of Sr-90 show a very low penetration depth (fig l) and tumours thicker than $4 \mathrm{~mm}$ were nevertheless successfully treated by treatment in two steps: after the initial treatment the tumour shrinks and after 6 months a second treatment is planned which reduces further the height of the tumour.

The major drawback of our Sr-90 applicator is its small size. Because uveal melanoma can have lateral extensions that are undetectable with ophthalmoscopy or ultrasonography and our applicator has only a limited diameter which rules out a safety margin of $2 \mathrm{~mm}$, we re-marked tumour growth on the border after several months. In these cases we had to re-treat borders with adjunctive methods such as xenon laser or diode laser; a second Sr-90 treatment was performed if the area was too large for laser.

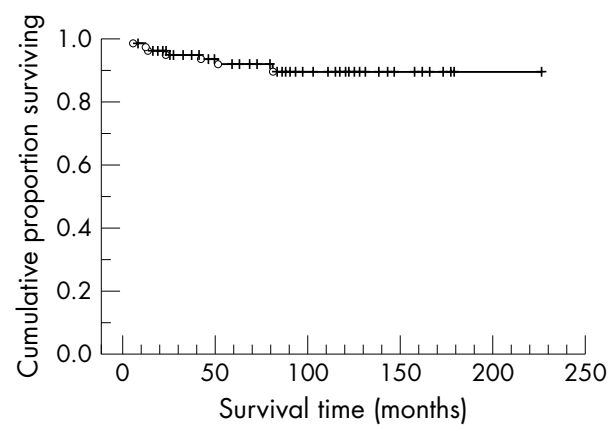

Figure 5 Kaplan-Meier survival curve of the eye after Sr-90 treatment, expressed in months of follow up, showing the high proportion of nonenucleated eyes. (Survival function: $\circ$, complete (enucleated); +, censored.)

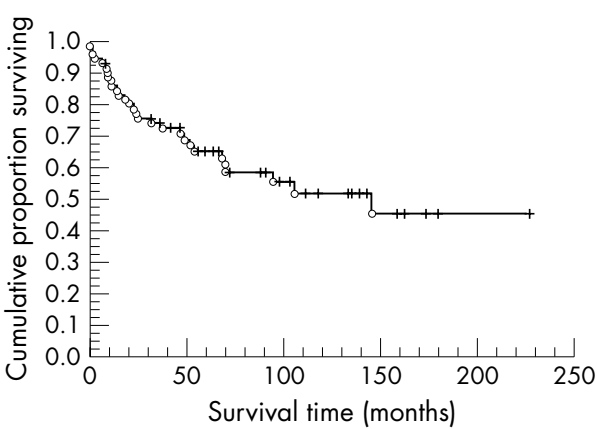

Figure 6 Kaplan-Meier estimates showing the proportion of patients retaining visual acuity of 1/10; expressed in months of follow up. All patients are included, also patients with initial pretreatment visual acuity less than $1 / 10$. (Survival function:, complete (visus after treatment $\leqslant 1 / 10) ;+$, censored.)

The actuarial ocular survival rate was $90 \%$ after 18 years. Only seven eyes had to be removed after Sr-90 treatment, with six eyes because recurrence on the border. ${ }^{8}$ If we had possessed a larger applicator this complication could have been eliminated. Because of the low penetration depth of Sr90 the complication rate is very low, no eyes were enucleated because of pain or neovascular glaucoma.

The final vision was mostly determined by the localisation of the tumour, with poor central vision if the tumour involved the macular region. After 15 years, $45 \%$ of patients had a minimum vision of $1 / 10$. We did not see visual impairment caused by radiation maculopathy or optic neuropathy, because of the limited penetration depth.

\section{Strontium-90 compared with iodine- 125}

Treatment with iodine-125 is popular, especially in North America, ${ }^{9-12}$ and because of the COMS trial ${ }^{1011}{ }^{13}$ the results are known. The dose rate of this $\gamma$ emitter is between $50 \mathrm{cGy} /$ hour and 100 cGy/hour, which is 100 times lower than Sr-90, and the application time varies between 30 and 300 hours. $^{2}$ The safety procedures around the penetrating $\gamma$ rays with the longer application time makes the procedure much more complex with more risks than Sr-90 treatment. The cost price of iodine treatment is much higher than Sr-90, owing to the frequent replacement of the iodine grains because of the very short half life of 60 days. The deeper penetration of the $\gamma$ rays allows treatment of larger and thicker tumours, but at the cost of causing more radiation damage to healthy surrounding tissues and, hence, optic neuropathy, maculopathy, and visual loss. ${ }^{14}$ In the medium sized arm of the COMS trial, $12.5 \%$ of eyes were enucleated and $10 \%$ were enucleated because of tumour recurrence during the first 5 years after treatment ${ }^{10}$; this is in the same range as our study.

If we compare the results on the visual acuity with studies of small and medium size tumours the rate of decline is much higher than in Sr-90 treated eyes. Nearly one half of the patients treated with iodine- 125 in the medium size tumour arm of the COMS lost substantial vision by only 3 years of 
Table 1 Visual acuity before treatment and after 3 years and 5 years

\begin{tabular}{llll}
\hline & \multicolumn{2}{l}{ Visual acuity } \\
\cline { 2 - 4 } Number of eyes & $\begin{array}{l}\text { Before } \\
\text { treatment }\end{array}$ & After $\mathbf{3}$ years & After $\mathbf{5}$ years \\
\hline $5 / 10-10 / 10$ & $55(62 \%)$ & $32(42 \%)$ & $20(38 \%)$ \\
$1 / 10-4 / 10$ & $20(22 \%)$ & $17(22 \%)$ & $13(25 \%)$ \\
Less than 1/10 & $13(15 \%)$ & $20(26 \%)$ & $13(25 \%)$ \\
HM, LP, no LP & $1(1 \%)$ & $7(9 \%)$ & $7(13 \%)$ \\
\hline HM, hand movements; LP, light perception. & & \\
\hline
\end{tabular}

follow up. ${ }^{10}$ Jones et al reported that only $33 \%$ of all patients retained vision of $1 / 10$ after 3 years. ${ }^{15}$ In the study of Wilson only $29 \%$ of patients had a final vision of $1 / 10$ of more after 45 months. ${ }^{5}$ In the study of Shields and starting with only those patients with initial good vision (more than 1/10), 69\% had poor vision ( $1 / 10$ or less) at 10 years of follow up. ${ }^{9}$ The vision decline continues also to progress with time: poor vision was seen in $1 \%$ of patients after 1 year, in $31 \%$ at 5 years, and in $69 \%$ at 10 years. ${ }^{9}$ In all these studies on small to medium sized tumours, the vision declines faster and with a much higher proportion of patients going legally blind than in our group, because of the deeper penetration of $\gamma$ radiation.

\section{Strontium-90 compared with ruthenium-106}

Ruthenium-106, a $\beta$ emitter, is used for small and medium sized tumours in Europe. ${ }^{46-20}$ All published studies incorporated also small tumours with a range between $10 \%$ and $20 \%$, which is similar to our study. The dose applied to the apex is between $60 \mathrm{~Gy}$ and $100 \mathrm{~Gy}$. The dose rate of ruthenium varies between $50 \mathrm{cGy} /$ hour and $100 \mathrm{cGy} /$ hour, which is hundred times less than Sr-90 (10200 CGy/hour) and this correlates with an application time of 1-7 days. Ruthenium has a low dose rate, which means that during radiotherapy repair of DNA destruction is possible; the high dose rate of Sr-90 excludes all repair during therapy. This lower dose rate makes it less effective than Sr-90. The recurrence rate is between $6 \%$ and $36 \%$ after $5-15$ years and the enucleation rate between $8 \%$ and $34 \%$ after $5-15$ years. ${ }^{17-22}$

The vision decline is slower than with iodine- 125 treatment, but is more pronounced than in strontium treated eyes. Loss of visual acuity to less than $1 / 10$ is reported to be between $62 \%$ and $81 \%$ after $10-15$ years; these studies do not differentiate between posterior and more anterior tumours. ${ }^{172123}$ Rouberol et al reported in their study on posterior tumours (as were our tumours), that only $19 \%$ of patients had a vision more than $1 / 10$ after 3 years. ${ }^{17}$ Only $22.7 \%$ retained good vision at the end and $20 \%$ of patients were enucleated. ${ }^{17}$ The complication rate of ruthenium treated eyes is lower than in iodine treated eyes, but is still much higher than in strontium treated eyes. ${ }^{17}{ }^{20}$ Radioinsensitivity (persistence of cycling cells) is described in ruthenium treated eyes, possibly related to the lower dose rates. ${ }^{24-27}$ Histology of the enucleated eyes after Sr-90 treatment showed tumour regrowth at insufficient treated borders, but the irradiated regions were tumour free. ${ }^{8}$

Because Sr-90 delivers extremely high doses in a short time, all the blood vessels in the treated area are closed in less than 14 days. $^{7}$ After a short swelling a shrinking process of the tumour sets in; the involution process is therefore much faster than in the other isotopes or proton treatment. ${ }^{6}{ }^{13} 17$ Melanoma cells are not radiosensitive and a tumour necrosis rather than therapeutic dose of radiation is required to destroy this tumour.

Survival rates of patients with Sr-90 treated eyes are similar to other treatments, a logical consequence of totally destroying the tumour. ${ }^{12} 1628-30$
The cost of the strontium applicator (our applicator was purchased in 1983 for $€ 1250$ ) should be far below all the other types because of the long half life; the treatment procedure should be safer because of the limited range of $\beta$ irradiation. Treatments should be completed more quickly because of the high dose rate. We regret that new applicators are not commercially available anymore, particularly plaques having a greater diameter than the one we used. Strontium plaques would enable brachytherapy of uveal melanomas be performed in centres that do not receive enough patients with this disease for other methods to be economically viable.

\section{ACKNOWLEDGEMENTS}

We thank Mrs M Bressinck for the help on the statistical program.

\section{Authors' affiliations}

R van Ginderdeuren, W Spileers, Department of Ophthalmology,

Kapucijnenvoer 33, UZ Leuven, B3000 Leuven, Belgium

E van Limbergen, Department of Radiotherapy, Gasthuisberg UZ

Leuven, B3000 Leuven, Belgium

Competing interests: none declared

\section{REFERENCES}

1 Damato BE. An approach to the management of patients with uveal melanoma. Eye 1993;7:388-97.

2 Pötter R, Van Limbergen E. Uveal melanoma. In: The GEC ESTRO handbook of brachytherapy. pp591-610.

3 Robertson DM. Changing concepts in the management of choroidal melanoma. Am J Ophthalmol 2003;136:161-70.

4 Lommatzsch PK. Radiotherapie der intraokularen Tumoren, insbesondere bei Aderhautmelanom. Klin Monatsbl Augenheilkd 1979;174:948-58.

5 Wilson MW, Hungerford JL. Comparison of episcleral plaque and proton beam radiation therapy for the treatment of choroidal melanoma. Ophthalmology 1999; 106:1579-87.

6 Egger E, Schalenbourg A, Zografos L, et al. Maximizing local tumor control and survival after proton beam radiotherapy of uveal melanoma. Int J Radiat Oncol Biol Phys 2001;51:138-47.

7 Missotten L, Dirven W, Van der Schueren A, et al. Results of treatment of choroidal malignant melanoma with high-dose-rate strontium-90 brachytherapy. Graefes Arch Clin Exp Ophthalmol 1998;236:164-73.

8 Parys-Van Ginderdeuren R, Van den Oord JJ, Missotten L. Results of histopathologic examination of three enucleated eyes with a choroidal melanoma afeter Strontium-90 irradiation. Bull Soc Belge Ophtalmol 1997;265:47-54.

9 Shields CL, Shields JA, Cater J, et al. Plaque radiotherapy for uveal melanoma. Long-term visual outcome in 1106 consecutive patients. Arch Ophthalmol 2000;1 18:1219-28.

10 Jampol L, Moy C, Murray T, et al. The COMS randomised trial of iodine-125 brachytherapy for choroidal melanoma. IV Local treatment failure and enucleation in the first 5 years after brachytherapy. COMS Report No 19. Ophthalmology 2002;109:2197-206

11 Collaborative Ocular Melanoma Study Group. Collaborative ocular melanoma study (COMS) randomised trial of I-125 brachytherapy for medium choroidal melanoma. Visual acuity after 3 years. COMS Report No 16. Ophthalmology 2001;108:348-66.

12 Char D, Kroll S, Phillips T, et al. Late radiation failures after iodine 125 brachytherapy of uveal melanoma compared with charged-particle (proton or helium ion) therapy. Ophthalmology 2002;109:1850-4.

13 Sieving PA. Fifteen years of work. The COMS outcomes for medium-sized choroidal melanoma. Arch Ophthalmol 2001;119:1067-8.

14 Packer S, Stoller S, Lesser ML, et al. Long-term results of iodine 125 irradiation of uveal melanoma. Ophthalmology 1992;99:767-73. 
15 Jones R, Gore E, Mieler W, et al. Posttreatment visual acuity in patients treated with episcleral plaque therapy for choroidal melanomas: dose and dose rate effects. Int J Radiation Oncology Biol Phys 2002;52:989-95.

16 Seregard S. Long-term survival after ruthenium plaque radiotherapy for uveal melanoma. A meta-analysis of studies including 1066 patients. Acta Opthalmol Scand 1999;77:414-17.

17 Rouberol F, Roy P, Kodjikian L, et al. Survival, anatomic, and functional longterm results in choroidal and ciliary body melanoma after ruthenium brachytherapy (15 years experience with beta-rays). Am J Ophthalmol 2004; 137:893-900.

18 Hungerford J. Current trends in the treatment of ocular melanoma by radiotherapy. Gregg Lecture 2001. Clin Experiment Ophthalmol 2003;31:8-13.

19 Seregard S, Trmpe E, Lax I, et al. Results following episcleral ruthenium plaque radiotherapy for posterior uveal melanoma. The Swedish experience. Acta Ophthalmol Scand 1997;75:11-16.

20 Summanen P, Immonen I, Kivela T, et al. Radiation related complications after ruthenium plaque radiotherapy of uveal melanoma. $\mathrm{Br} J$ Ophthalmol 1996:80:732-9.

21 Lommatzsch PK. Results after $\beta$ irradiation ( ${ }^{106} \mathrm{Ru}^{-106} \mathrm{Rh}$ ) of choroidal melanomas: 20 years' experience. Br J Ophthalmol 1986;70:844-51.

22 Guthoff R, von Domarus D, Steinhorst U, et al. 10 Jahre Erfahrung mit der Ruthenium-106/Rhodium 106-Behandlung des malignen Melanoms der Aderhaut - Bericht über 264 bestrahlte Tumoren. Klin Monatsbl Augenheilkd 1986;188:576-83.
23 Summanen P, Immonen I, Kiveal T, et al. Visual outcome of eyes with malignant melanoma of the uvea after ruthenium plaque radiotherapy. Ophthalmic Surg Lasers 1995;26:449-60.

$24 \mathrm{Pe}^{\prime}$ er J, Stafani FH, Seregard S, et al. Cell proliferation activity in posterior uveal melanoma after Ru-106 brachytherapy: an EORTC ocular oncology group study. Br J Ophthalmol 2001;85:1208-12.

25 Klaus H, Lommatzsch PK, Fuchs U. Histopathology studies in human malignant melanomas of the choroid after unsuccessful treatment with ${ }^{106} \mathrm{Ru} /{ }^{106} \mathrm{Rh}$ ophthalmic applicators. Graefes Arch Clin Exp Ophthalmol 1991;229:480-6.

26 Messmer E, Bornfeld N, Foerster M, et al. Histopathologic findings in eyes treated with a ruthenium plaque for uveal melanoma. Graefes Arch Clin Exp Ophthalmol 1992;230:391-6.

27 Seregard S, Lundell G, Lax I, et al. Tumour cell proliferation after failed ruthenium plaque radiotherapy in posterior uveal melanoma. Acto Ophthalmol Scand 1997:75:148-54

28 Kujala E, Makitie T, Kivela T. Very long term prognosis of patients with malignant uveal melanoma. Invest Ophthalmol Vis Sci 2003;44:4651-9.

29 Collaborative Ocular Melanoma Study Group. The COMS randomised trial of lodine 125 brachytherapy for choroidal melanoma, III: Initial mortality findings. COMS Report No 18. Arch Ophthalmol 2001;1 19:969-82.

30 Straatsma B, Diener-West M, Caldwell R, et al. Mortality after deferral of treatment or No treatment for choroidal melanoma. Am J Ophthalmol 2003; 136:47-54. 